\title{
Associations between neurological and rheumatological manifestations in vitamin D deficiency and vitamin D \\ levels
}

\section{Faiza A. Qari ${ }^{1}$, Tariq A. Nasser ${ }^{2}$}

Objective: To investigate the associations between the neurological and rheumatologic manifestations of vitamin D deficiency a $(\mathrm{OH}) \mathrm{D}$ ] and laboratory values of bone profile level and levels of 25-hydroxyvitamin D [25].

Methodology: We conducted a case series study in patients with osteomalacia who were followed up at King Abdulaziz Medical City, Jeddah, between January 2010 and December 2011. We collected patients' demographic data and gathered information on etiological factors for vitamin D deficiency as well as clinical presentations (typical and neurological with muscular) and radiological findings. The t-test was used to determine whether there were associations between the neurological manifestations of vitamin D deficiency and vitamin D levels and bone profile. A $P$-value $<0.05$ was considered significant.

Results: Sixty patients were enrolled in the study. A $73 \%$ neurological presentation, like progressive muscle weakness, and proximal weakness was observed more often than distal weakness. In addition, gait disturbances were observed in $61.7 \%$ of all patients with neurological presentations. There were no significant associations between neurological rheumatological manifestations and bone profile or vitamin D levels. There were significant associations between difficulty in walking and the levels of serum calcium and phosphate $(P=0.043$ and $P=0.037$, respectively). 
Conclusion: Neurological rheumatological manifestations of vitamin D deficiency are not associated with the $25(\mathrm{OH}) \mathrm{D}$ levels or laboratory values of bone profile level.

Table-I: Laboratory data of the study subjects.

\begin{tabular}{|l|c|c|c|}
\hline \multicolumn{1}{|c|}{ Laboratory test levels* } & Mean \pm SD & Minimum & Maximum \\
\hline Calcium & $2.001 \pm 0.21$ & 1.19 & 2.39 \\
\hline Phosphate & $2.14 \pm 7.7$ & 0.35 & 4.4 \\
\hline Magnesium & $0.77 \pm 0.142$ & 0.45 & 1.30 \\
\hline Creatinine & $76.85 \pm 52$ & 40 & 432 \\
\hline HCO $_{3}$ & $22 \pm 2.55$ & 16 & 28 \\
\hline Vitamin D3 & $18.6 \pm 22.54$ & 10 & 79 \\
\hline
\end{tabular}

Abbreviations: $\mathrm{HCO}_{3}$, bicarbonate; $\mathrm{PTH}$, parathyroid hormone

*Reference range for laboratory tests: calcium $(2.12-2.52 \mathrm{mmol} / \mathrm{L})$; creatinine $(53-115 \mu \mathrm{mol} / \mathrm{L})$; $\mathrm{HCO}_{3}(21-28 \mathrm{mmol} / \mathrm{L})$; magnesium $(0.7-1.0 \mathrm{mmol} / \mathrm{L})$; phosphate $(0.81-1.5 \mathrm{mmol} / \mathrm{L})$; vitamin D3 (75-200 nmol/L). PTH (11 - $54 \mathrm{pg} / \mathrm{mL})$.

Table-II: Associations between 25(OH) D levels and bone profile with the presence or absence of atypical neurological presentations in study subjects*

\begin{tabular}{|c|c|c|c|c|c|c|c|c|c|c|c|c|}
\hline \multirow{2}{*}{ Character } & \multicolumn{3}{|c|}{ Paresthesia } & \multicolumn{4}{|c|}{ Tetany } & \multicolumn{3}{c|}{ Difficulty in } & \multicolumn{3}{|c|}{ Deformity } \\
\cline { 2 - 11 } & + & - & $\begin{array}{c}\mathrm{T} \\
\text { test }\end{array}$ & + & - & $\begin{array}{c}\mathrm{T} \\
\text { test }\end{array}$ & + & - & $\begin{array}{c}\text { T } \\
\text { test }\end{array}$ & + & - & $\begin{array}{c}\text { T } \\
\text { test }\end{array}$ \\
\hline $\begin{array}{c}\text { Calcium } \\
\text { level } \\
\text { mmol/L }\end{array}$ & 2.1 & 2.0 & $\mathbf{0 . 9}$ & 1.89 & 2.01 & $\mathbf{0 . 5 4}$ & 2.00 & 2.0 & $\mathbf{0 . 0 4 3}$ & 2.0 & 2.00 & $\mathbf{0 . 6}$ \\
\hline $\begin{array}{c}\text { Phosphate } \\
\text { mmol/L }\end{array}$ & 0.79 & 0.7 & $\mathbf{0 . 2 9}$ & 0.69 & 0.7 & $\mathbf{0 . 9 7}$ & 0.65 & 0.7 & $\mathbf{0 . 0 3 7}$ & 0.7 & 0.7 & $\mathbf{0 . 5}$ \\
\hline $\begin{array}{c}\text { Magnesium } \\
\text { mmol/L }\end{array}$ & 0.8 & 0.8 & $\mathbf{0 . 4 2}$ & 0.81 & 0.8 & $\mathbf{0 . 3 2}$ & 0.73 & 0.8 & $\mathbf{0 . 2}$ & 0.9 & 0.8 & $\mathbf{0 . 3}$ \\
\hline $\begin{array}{c}\text { Alkaline } \\
\text { phosphatase } \\
\text { ul/L }\end{array}$ & 350 & 472 & $\mathbf{0 . 9 1}$ & 334 & 375 & $\mathbf{0 . 3 6}$ & 350 & 351 & $\mathbf{0 . 8}$ & 386 & 351 & $\mathbf{0 . 6}$ \\
\hline $\begin{array}{c}\text { Vitamin D3 } \\
\text { level } \\
\text { nmol/L }\end{array}$ & 10 & 10 & $\mathbf{0 . 8 9}$ & 17 & 10 & $\mathbf{0 . 2 1}$ & 10 & 10 & $\mathbf{0 . 3 2}$ & 10 & 10 & $\mathbf{0 . 3}$ \\
\hline $\begin{array}{c}\text { PTH level } \\
\text { pg/ml }\end{array}$ & 268 & 247 & $\mathbf{0 . 9 2}$ & 287 & 248 & $\mathbf{0 . 5 2}$ & 287 & 286 & $\mathbf{0 . 6 2}$ & 304 & 286 & $\mathbf{0 . 1}$ \\
\hline
\end{tabular}

*The signs (+) and (-) denote the presence and absence of neurological clinical manifestations in correlation with laboratory level values of bone parameters (calcium, phosphate, magnesium, alkaline phostase) and level of vitamin D3 and PTH 1.

T-test showed if there is a significant correlation between the neurological manifestations with a level of laboratory values of bone profile and vitamin D3 level

Reference range for laboratory tests: alkaline phosphatase $(50-130 \mathrm{ul} / \mathrm{L})$; calcium $(2.12-2.52$ $\mathrm{mmol} / \mathrm{L})$; creatinine $(53-115 \mu \mathrm{mol} / \mathrm{L}) ; \mathrm{HCO}_{3}(21-28 \mathrm{mmol} / \mathrm{L})$; magnesium $(0.7-1.0 \mathrm{mmol} / \mathrm{L})$; 
phosphate $(0.81-1.5 \mathrm{mmol} / \mathrm{L})$; PTH (1.6 -6.9 pg/mL); vitamin D3 (50-125 nmol/L). PTH (11 $54 \mathrm{pg} / \mathrm{mL}$ ) 